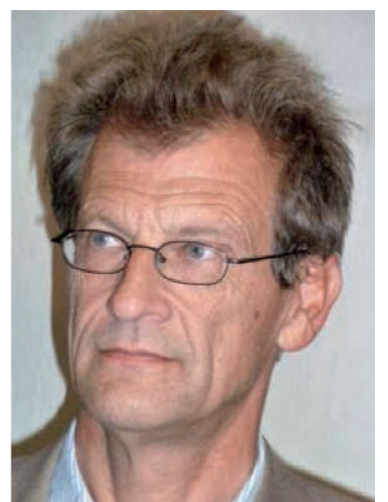

Prof. Dr. med. Dr. rer. nat. Bernhard Uehleke, Zürich/Berlin

\section{Traditionelle Europäische Medizin (TEM) und Traditionelle Europäische Naturheilkunde (TEN) - brauchen wir mehrere Begriffe?}

Als sich vor etlichen Jahren das Interesse in der Komplementärmedizin immer mehr den asiatischen Heilverfahren zuneigte - wobei deren Anhänger deren Jahrtausende umfassende Traditionslinien strapazierten -, wiesen manche Naturheilkundler auf eine Rückbesinnung auf unsere eigenen Wurzeln hin. Ähnlich dem System der 5 Wandlungsphasen in der Traditionellen Chinesischen Medizin (TCM) oder den 3 Doshas in der Ayurveda haben wir mit der auf den 4 Elementen aufbauenden Viersäftelehre schliesslich auch ein interessantes Modell, das weit über 2000 Jahre vergleichsweise recht geradlinig den Kern unserer westlichen Medizingeschichte darstellte.

Warum sollten wir nicht unsere eigene Tradition wieder stärker berücksichtigen, zumal etliche Aspekte der Humoralpathologie ja noch tief in der mitteleuropäischen Volksheilkunde, ja, sogar in modernen Konstitutionslehren verankert sind? Während einer Reise in die Mongolei zur Begutachtung der dort propagierten Traditionellen Mongolischen Medizin (TMM) (die sich freilich die meisten ihrer Vorstellungen und Verfahren aus der TCM entlehnt hat) wurde den Teilnehmern dann klar: Wir brauchen auch etwas Entsprechendes in Europa! Inhaltlich geht es bei uns dann entsprechend um die Hauptlinien der europäischen (oder westlichen) Medizin, insbesondere mit den Aspekten der Humoralpathologie, wobei man die Verfahren beim einzelnen Patienten schon an die modernen Erkenntnisse anpassen sollte. Der Begriff Traditionelle Deutsche Medizin scheidet nach dem während der Zeit des Nationalsozialismus proklamierten Konzepts «Neue deutsche Medizin» direkt aus und wäre auch zu eng gefasst, da die Wurzeln der Humoralpathologie ja in der Antike liegen und im alten Griechenland und Italien (Rom) sowie in den angrenzenden Ländern zu verorten sind. Übrig bleibt dann der Bezug auf das Europäische oder auf das Westliche. Der zweite Begriff - Traditionelle Westliche Medizin (TWM) - könnte mit der Medizin der Indianer aus Nordamerika verwechselt werden; somit schien uns damals der Begriff Traditionelle Europäische Medizin (TEM) geeigneter.

Freilich war zunächst nicht klar, was der Inhalt einer TEM sein sollte. Etliche Kollegen aus der Naturheilkunde bzw. Komplementärmedizin waren der Meinung, schon wieder ein neuer Begriff störe die Entwicklung und insbesondere die soeben begonnene Akademisierung bzw. Etablierung der Naturheilkunde an den ersten Universitäten. Ein entsprechender Delphi-Prozess zum Begriff und zu dessen Inhalten wurde zunächst teilweise abgelehnt und dann durch fadenscheinige «fachpolitische» Argumente der Fachgutachter von etlichen Zeitschriften abgelehnt, bevor die Ergebnisse schliesslich in dieser Zeitschrift veröffentlicht wurden [1].

Einen Vorteil des neuen Begriffs TEM sahen wir damals vor allem darin, dass er den schlecht definierten und meist mehrdeutig verwendeten Begriff Naturheilkunde ablösen hätte können. Die Naturheilkunde hatte sich ja gegenüber den klassischen Naturheilverfahren zunehmend erweitert. Dabei wurden auch einige ehemals bei der Etablierung der Naturheilkundebewegung im 19. Jahrhundert eindeutig zur «Schulmedizin» gehörende Verfahren wie Aderlass und Blutegeltherapie, aber auch balneologische Verfahren usw. einbezogen, nachdem diese von der modernen wissenschaftlichen Medizin aufgegeben wurden, und analog dann sogar - wenn auch nicht ganz unumstritten technische Verfahren aus der «Schulmedizin» des frühen 20. Jahrhunderts wie Eigenblut, Ozontherapie usw. Mit der TEM könnte man solche Verfahren, die ja weder der «Natur» noch der «naturgemässen Lebensweise» entsprechen, ganz zwanglos eingliedern, solange es sich um eher unspezifische Reiz-Therapien handelt, die möglicherweise auf die «Säftezusammensetzung» Einfluss nehmen können. Eine Einordnung als Naturheilverfahren ist aber sehr problematisch. Eine Mehrheit der an unserem Delphi-Prozess beteiligten Fachleute zählte übrigens besonders spezielle Verfahren wie Homöopathie nicht zur TEM, weil

$\begin{array}{ll}\text { KARGER } & \text { @ 2013 S. Karger GmbH, Freiburg } \\ \text { 1015-0684/13/0254-0201\$38.00/0 } \\ \begin{array}{l}\text { Fax +49 761 452 07 14 } \\ \begin{array}{l}\text { Information@Karger.com } \\ \text { www.karger.com }\end{array}\end{array} & \begin{array}{l}\text { Accessible online at: } \\ \text { www.karger.com/szg }\end{array}\end{array}$


diese nicht im Rahmen der europäischen Entwicklungsstränge liegen, sondern sehr eigene Vorstellungen entwickelt haben, die umgekehrt auch keinerlei Einfluss auf den weiteren Verlauf der Medizinentwicklung hatten.

Schweizer Heilpraktiker verwenden unabhängig von diesen Ansätzen etwa ebenso lange den Begriff Traditionelle Europäische Naturheilkunde (TEN) - ohne diesen bisher genauer zu definieren. Dies mag damit zusammenhängen, dass der Terminus Medizin zunehmend für die offiziellen Ärzte vorbehalten wird. Auch die neuesten internationalen Entwicklungen, bei denen die nichtärztlichen Heiler (non-medical healers) inzwischen fast durchgängig von "healing» oder "health care» anstelle von «medicine» sprechen, zeigen dies auf. So kennt man neuerdings statt CAM (Complementary and Alternative Medicine) auch $\mathrm{CAH}$ (Complementary and Alternative Healing) (oder CAHC - Complementary and Alternative Health Care) oder statt Integrative Medicine auch IC (Integrative Healing) oder IHC (Integrative Health Care).

Erst vor Kurzem wurde deutlich, dass diese Heilpraktiker den Bogen für die TEN wesentlich weiter spannen und dabei schlichtweg alle Heilverfahren, die irgendeinen Bezug zu Europa haben, aufgreifen. Damit gehen sie über die Naturheilverfahren im engeren Sinne weit hinaus. $\mathrm{Zu}$ dem werden dabei recht weitgehende, beispielsweise an Esoterik anmutende Verknüpfungen gebildet. Zur Klarheit würde beitragen, wenn dann wenigstens der klarere und umfassendere Begriff Komplementärmedizin verwendet würde. Eine Traditionelle Europäische Komplementärmedizin könnte sogar die World Health Organization (WHO) interessieren, die ja bisher für die westliche Welt kein anderen Regionen entsprechendes Konzept einer traditionellen regionalen Medizin gegenüberstellen konnte, weil sie die Bedeutung der TEM über die westliche $\mathrm{Me}$ dizingeschichte hinaus nicht erkannt hat. Da es aber den Begriff und das Konzept der CAM in der westlichen Welt erst seit rund zwei Jahrzehnten gibt, könnte man einwenden, dass sich dieses nicht gut mit einer Tradition vereinbaren lässt. Der Kürze zuliebe sollte dabei aber trotzdem klar sein, dass mit einer Traditionellen Europäischen Kom- plementärmedizin eigentlich eine Sammlung von denjenigen komplementärmedizinischen Verfahren gemeint ist, die aus europäischen Entwicklungen entstanden sind und/ oder anhand der traditionellen Modelle und Sichtweisen in der Medizin erklärt und praktiziert werden.

Die den positiven Aspekt der «Natur» missbrauchenden Anhänger einer breit umfassenden TEN mögen solche Überlegungen als kleinlich betrachten. Ich halte es aber für unabdingbar, dass klare Begriffe und Definitionen benutzt werden, um die Anschlussfähigkeit der Komplementärmedizin mit ihrem weitgefächerten Angebot an Verfahren und Anbietern an die wissenschaftliche Medizin nicht zu gefährden. Unabhängig aber von den Begriffen sollte man bei einem medizinischen Bezug zur Humoralpathologie mit den entsprechenden Konstitutionen usw. auch endlich daran gehen, die medizinische Relevanz dieses Modells und seiner Konsequenzen für die Praxis durch neue Studien zu erfassen. Hierzu sind zwar einige der ausleitenden Verfahren wie Aderlass oder Blutegeltherapie untersucht worden, aber es wurden keine Untergruppen nach Konstitutionen oder humoralpathologischen Befunden betrachtet. Wenn diese Verfahren in der Praxis und in Studien aber für alle oder die meisten Patienten unabhängig von der Konstitution usw. wirken, dann könnte man auf den Ballast der traditionellen Modelle möglicherweise verzichten (wie sich dies teilweise bei der westlichen Akupunktur zur Schmerzbehandlung abzeichnet).

Zusammenfassend möchte ich mit diesem Beitrag zum kritischen Umgang mit Definitionen und Begrifflichkeiten der Naturheilkunde und zur Forschung über humoralpathologische Grundlagen aufrufen. Eine gemeinsame Positionierung von Heilpraktikern, Ärzten und Forschern würde die Durchsetzung von traditionellen europäischen Heilverfahren erheblich erleichtern.

\section{Literatur}

1 Uehleke B: Naturheilverfahren und «Traditionelle Europäische Medizin» TEM: Ergebnisse einer Experten-Umfrage (Delphi-Methode). Schweiz Zschr Ganzheitsmedizin 2007;19:199-203. 